\title{
APPLICATION OF 3D TREE MODELING USING POINT CLOUD DATA BY TERRESTRIAL LASER SCANNER
}

\author{
R. Kumazaki ${ }^{1, *}$, Y. Kunii $^{1}$ \\ ${ }^{1}$ Department of Landscape Architecture Science, Tokyo University of Agriculture, 1-1-1 \\ Sakuragaoka, Setagaya, Tokyo, 156-8502, Japan (blank.generation.charlie@gmail.com)(y3kunii@nodai.ac.jp)
}

Commission III, WG III/10

KEY WORDS: Japanese Garden, tree point cloud data, 3D tree model, TLS-QSM method, reproducibility

\begin{abstract}
:
Constructing 3D models for trees such as those found in Japanese gardens, in which many species exist, requires the generation of tree shapes that combine the characteristics of the tree's species and natural diversity. Therefore, this study proposes a method for constructing a 3D tree model with highly-accurate tree shape reproducibility from tree point cloud data acquired by TLS. As a method, we attempted to construct a 3D tree model using the TreeQSM, which is open source for TLS-QSM method. However, in TreeQSM, since processing is based on the assumption that the tree point cloud consists of data related to trunks and branches, measuring trees in which leaves have fallen is recommended. To solve this problem, we proposed an efficient classification process that mainly uses thresholds for deviation and reflectance, which are the adjunct data of the object that can be acquired by laser measurement. Furthermore, to verify accuracy of the model, position coordinates from the constructed 3D tree model were extracted. The extracted coordinates were compared with the those of the tree point cloud data to clarify the extent to which the 3D tree model was constructed from the tree point cloud data. As a result, the 3D tree model was constructed within the standard deviation of $0.016 \mathrm{~m}$ from the tree point cloud data. Therefore, the reproducibility of the tree shape by the TLS-QSM method was also effective in terms of accuracy.
\end{abstract}

\section{INTRODUCTION}

\subsection{Background}

Studies that accurately records the shape information of garden trees are insufficient even considering previous case studies of garden trees. There have been many studies that attempted to restore past garden plantings, but these are based on drawings, photographs, literature surveys, and drawing descriptions using picture scrolls, and there is no material that records the tree shape of that era in detail. From this, restoring the landscape of a Japanese garden by past planting has been a speculative attempt (Tomoki, 2011)(Taketoshi, 1984)(Taketoshi, 1994).

Surveying Japanese gardens began in 1919 when laws to preserve historic sites, scenic spots, and natural monuments were enacted. In August 1923, as a representative initiative, Hiroshi Hara conducted flat plate surveying and drafting and surveyed about 60 gardens including Kinkaku-ji temple. Since then, flat plane surveying has long been used as a surveying method for plotting Japanese gardens, and the need to record actual measurements is high due to the importance of cultural value of Japanese gardens. However, recording tree shape information using flat plate surveying is very limited. Therefore, in thid study, we will use terrestrial laser scanner (TLS) as a means to record accurate information on tree shapes and verify its practical effectiveness.

\subsection{Purpose}

In previous studies, there have been few analytical studies using laser measurement technology for trees, and among them, even fewer studies that considered the means to grasp the shape of individual trees in detail. The reason is that trees have a complicated geometrical shape (Jucker, 2017)(Zhang, 2014)(Livny, 2010), which presents significant obstacles in data analysis. For this reason, it has been extremely difficult to process tree point cloud data, extract specific parts such as trunks and branches, and visualize tree shapes. Furthermore, constructing 3D models for trees such as those found in Japanese gardens, in which many species exist, requires the generation of tree shapes that combine the characteristics of the tree's species and natural diversity (Yasuhiro, 2006). Therefore, we focused on reconstructing tree shape characteristics, and considered the importance of 3D tree models of garden trees in Japanese gardens. In view of the above, this study proposes a method for constructing a 3D tree model with highly-accurate tree shape reproducibility from tree point cloud data using the TLS-QSM method.

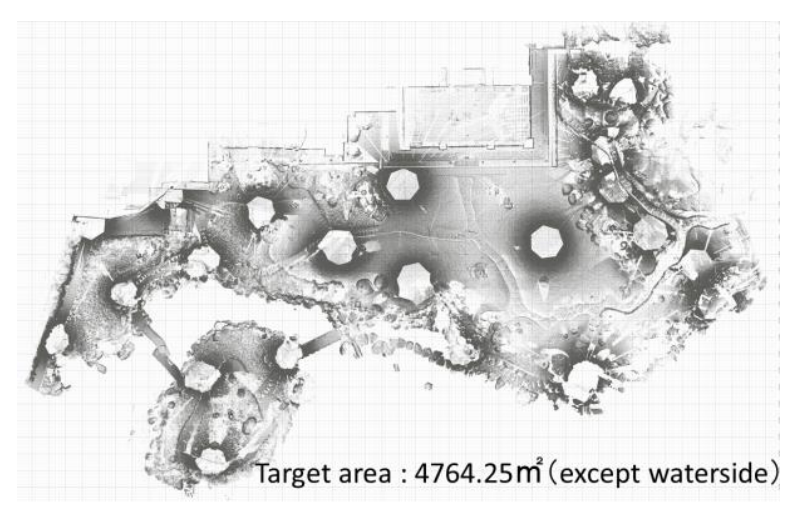

Figure 1. Target area for constructing 3D tree models

\footnotetext{
* Corresponding author
} 


\section{CONSTRUCTION OF JAPANESE GARDEN 3D TREE MODEL}

A measurement survey using the TLS (VZ-400i) was conducted at Kiyosumi Garden from May 29-31, 2019 and June 1,2 , and 4, 2019, for a total of six day. The appointed place for constructing $3 \mathrm{D}$ tree models in the garden was around the Taisho Memorial Hall, which is a major building in Kiyosumi Garden. (Figure 1).

\subsection{Construction of 3D Tree Model by TLS-QSM Method}

Figure 2 shows the process from the measurement in a Japanese garden using TLS to the construction of a 3D tree model. The TLS-QSM method used in this study is a method in

MEASUREMENT
(1) Surveying at Kiyosumi Garden
(2) Registration

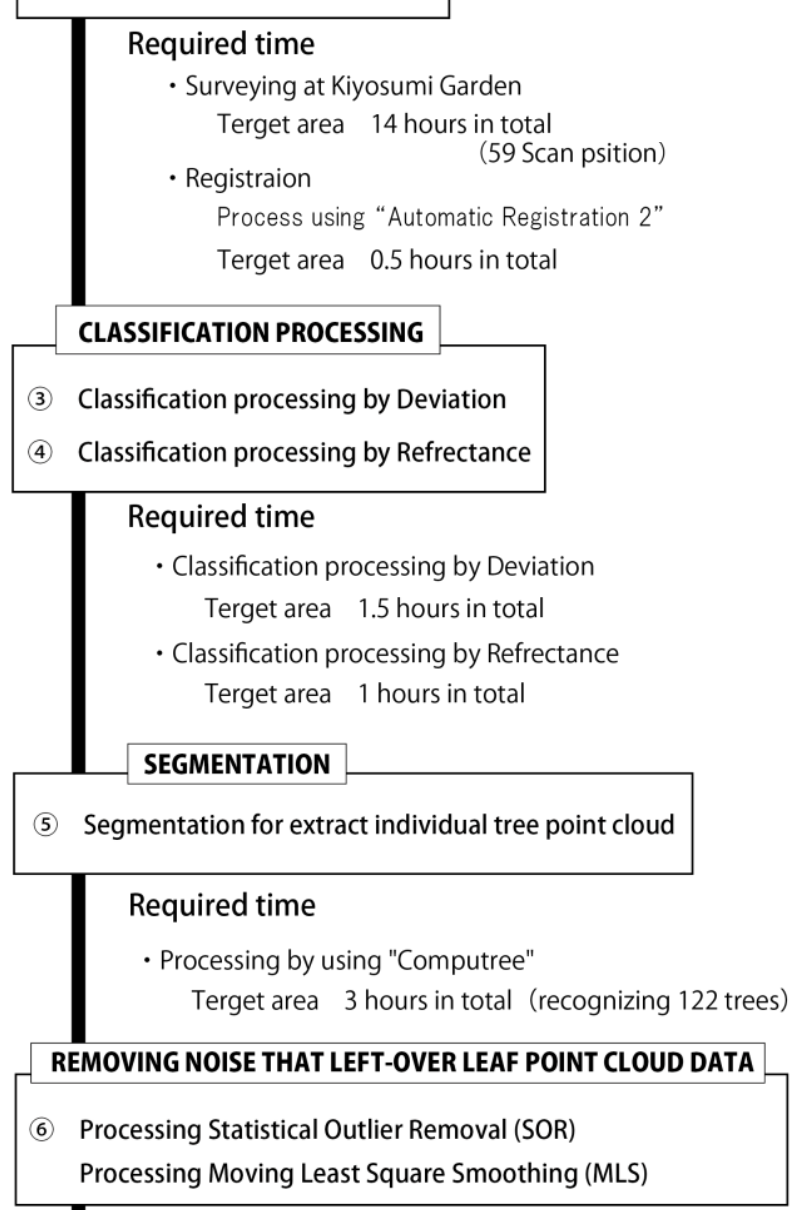

Required time

Terget area 1.5 hours in total

\section{CONSTRUCTION OF 3D TREE MODEL}

(7) Construction of 3D tree model by TLS-QSM method

\section{Required time}

Terget area 5 hours in total (construct 105 trees)

Figure 2. Construction process of 3D tree model

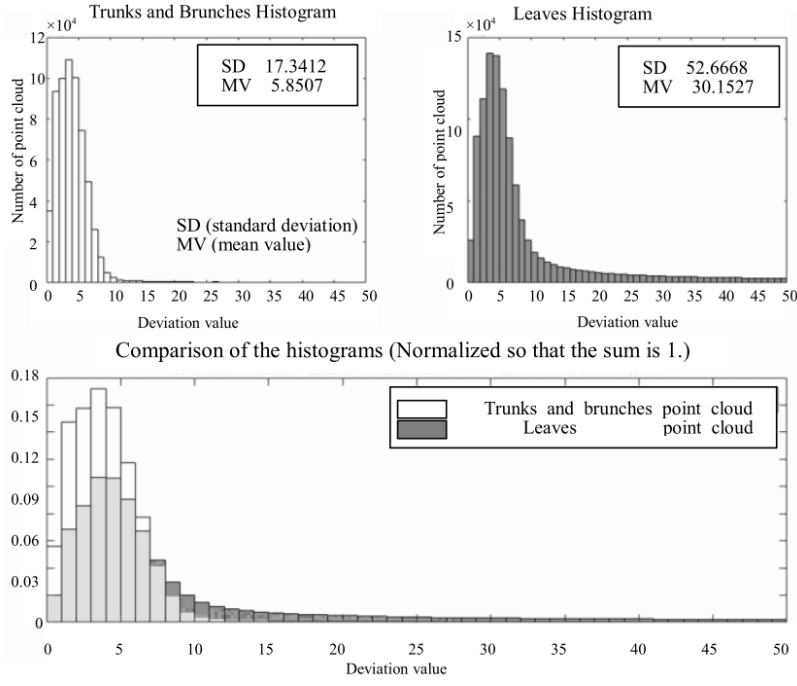

Figure 3. Deviation histogram

which a tree point cloud obtained by a TLS is divided into segments, with the radius and direction of each segment locally approximated and reconstructed as a continuous cylindrical model. By using this method, it is possible to construct a 3D tree model with small branch details that are difficult to generate automatically (Åkerblom, 2015)( Åkerblom, 2017)( Raumonen, 2013). In this study, we attempted to construct a 3D tree model using the open-source method "TreeQSM" (Inverse Tampere, 2019).

2.1.1 Classification of Leaves Point Cloud and Trunks and Branches Point Cloud: In TreeQSM, since processing is based on the assumption that the tree point cloud consists of data related to trunks and branches, measuring trees in which leaves have fallen is recommended (Raumonen, 2017). However, there are various tree species in Japanese gardens, and it is necessary to propose a method to classify point clouds consisting of leaves and those consisting of trunks and branches when applying TreeQSM without restricting tree species. To solve this problem, we proposed an efficient classification process that mainly uses thresholds for deviation and reflectance (RIEGL, 2017)(Calders, 2017), which are the adjunct data of the object that can be acquired by laser measurement.

Therefore, a histogram analysis of the deviation and reflectance was performed to confirm an effective threshold for the deletion of leaves point cloud. As a result, it was confirmed that the deviations of the trunks and branches point cloud were often distributed in values of 5 or less (Figure 3). Furthermore, as a result of the analysis of the reflectance values, it was confirmed that most of the reflectance values of the trunks and branches point cloud, regardless of the tree species, were distributed in the range of $-7.0 \mathrm{~dB}$ to $-5.5 \mathrm{~dB}$ or more (Figure 4). Therefore, we set thresholds for deviation and reflectance, and the results of the extraction process of the trunks and brunches point cloud are shown in Figure 5.

2.1.2 Segmentation Process for Extracting Single Tree Data: TreeQSM used in this study is based on the fact that the tree point cloud to be processed are composed of single tree data. Therefore, it is necessary to perform the segmentation process to recognize each tree point cloud as an individual data. In this study, the free software "Computree" (Hackenberg, 2015) (Computree group, 2010) was used to perform the 


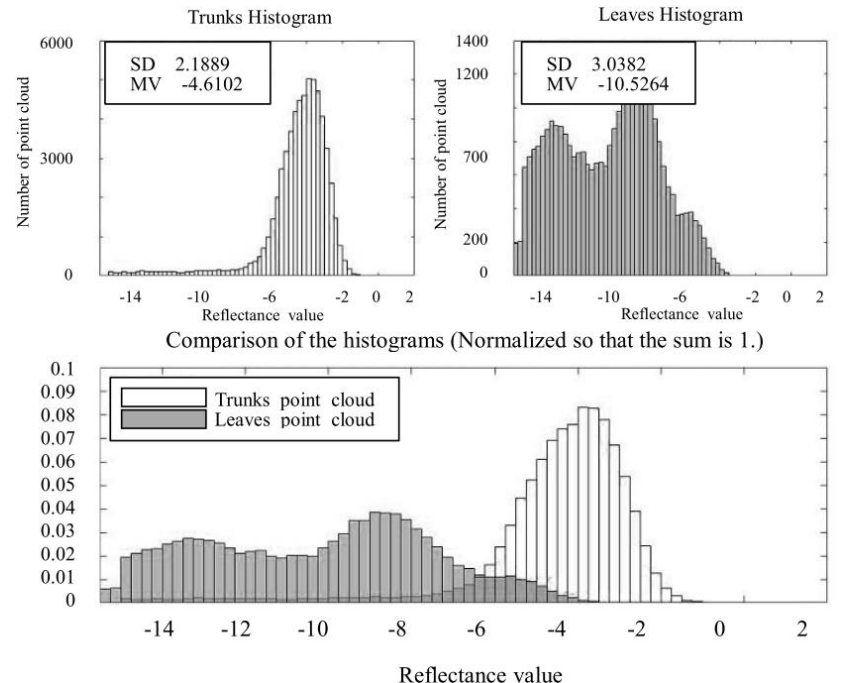

(a) Black pine

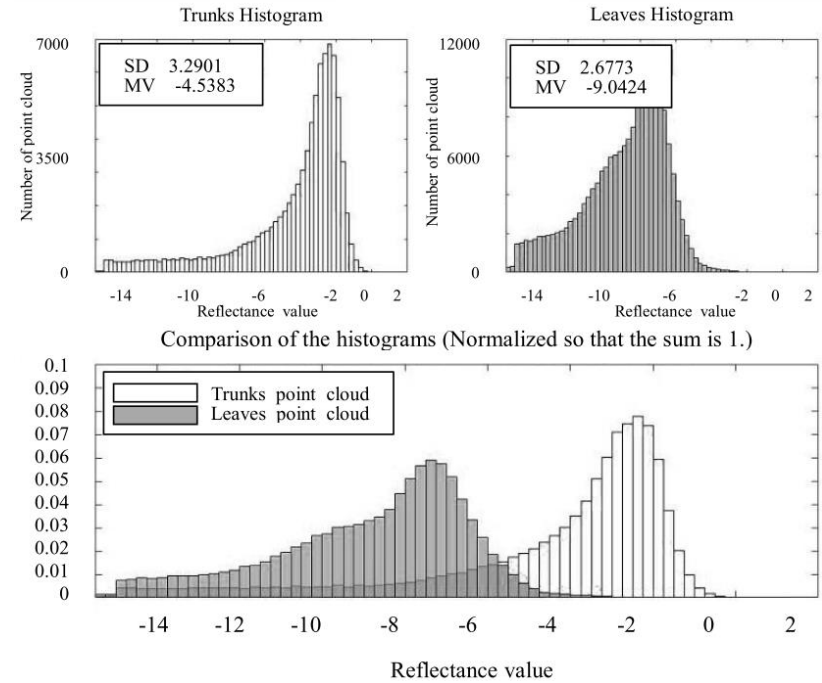

(c) Machilus thunbergii

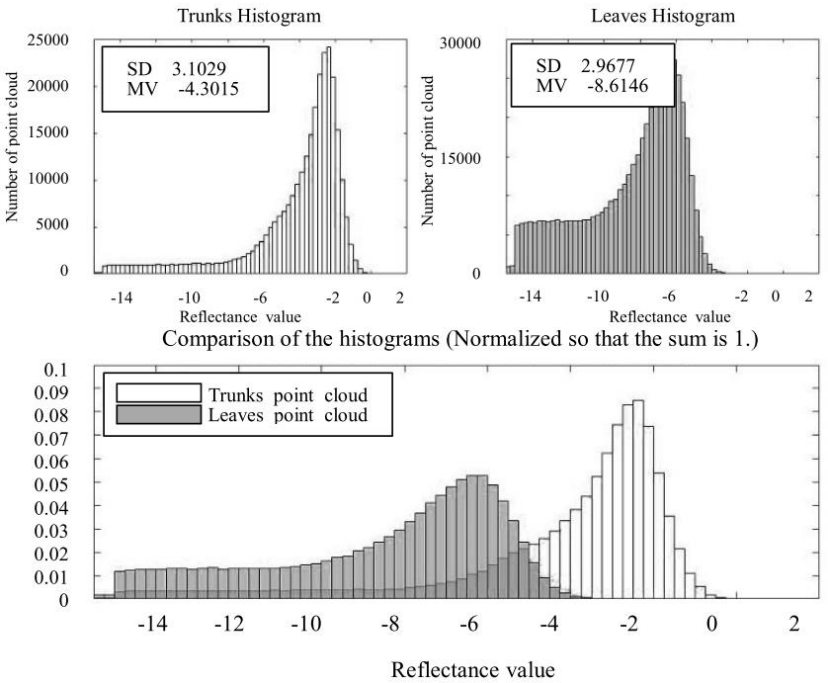

(b) Camphor
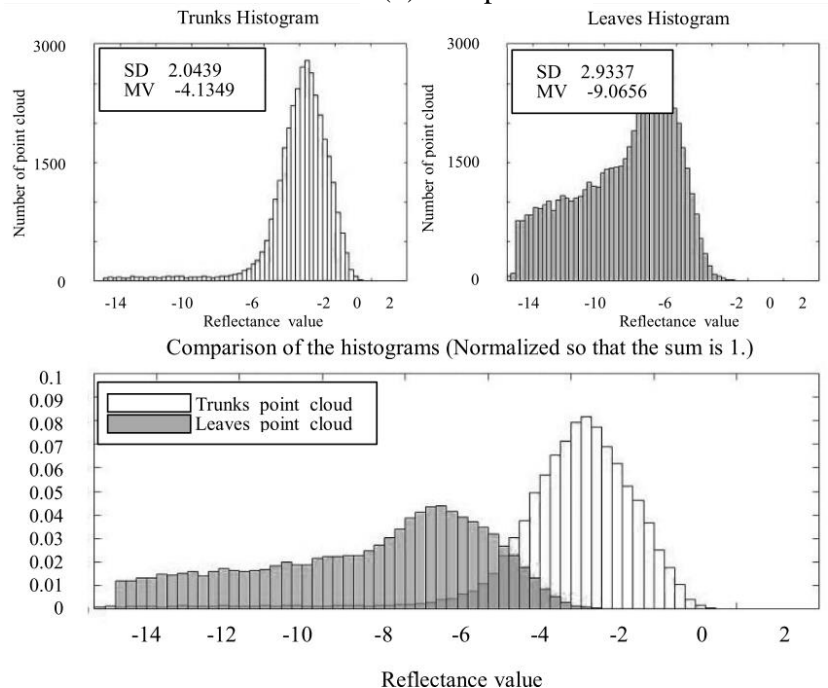

(d) Japanese wax tree

Figure 4. Reflectance histogram of each tree species
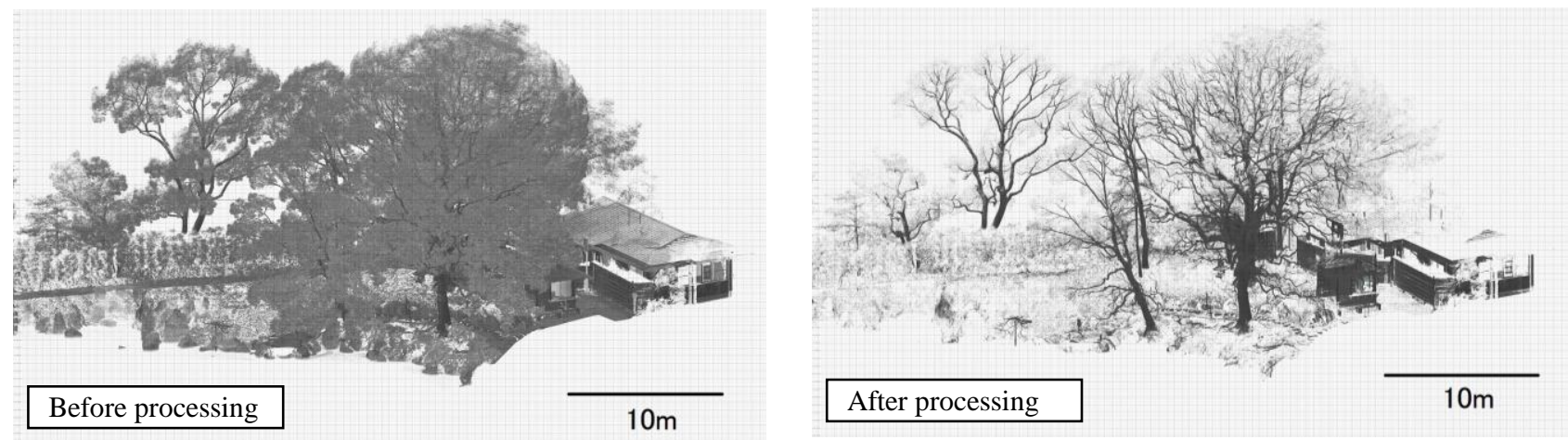

Figure 5. Classification using deviation and reflectance

segmentation process of the tree point cloud. The result of the segmentation process by Computree is shown in Figure 6.

\subsubsection{Noise Processing of the Remaining Leaves Point} Cloud: The effectiveness of the classification of leaves point cloud and trunks and branches point cloud using information on deviation and reflectance was demonstrated. However, when the leaves point cloud is removed using deviation and reflectance, some unwanted point cloud is left as noise. If the noise is not completely removed, there is a concern about the effect on the reproducibility of the 3D tree model constructed by the TreeQSM. Therefore, we used Statistical Outlier Removal (SOR) and Moving Least Square Smoothing (MLS) to noise processing (Figure 7). 


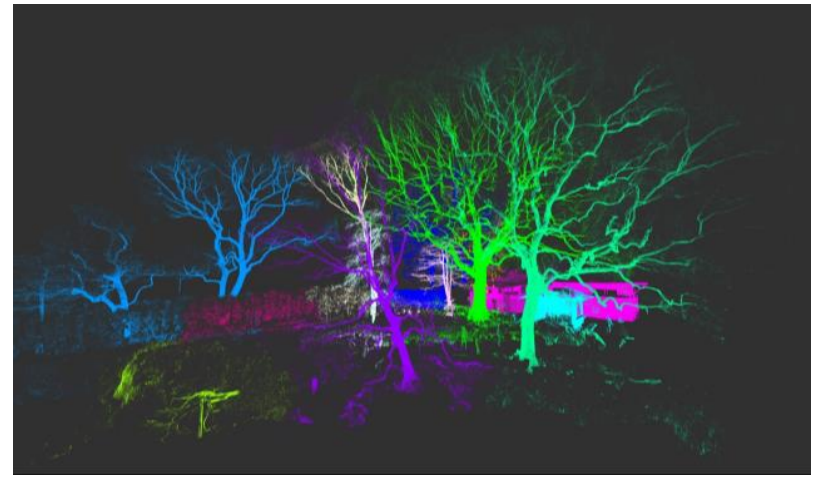

Figure 6. Segmentation using Computree

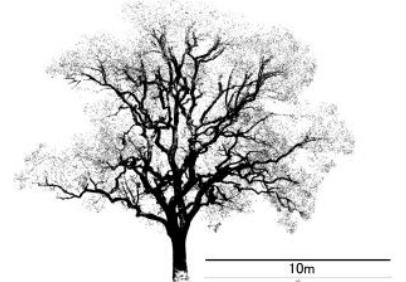

Before processing

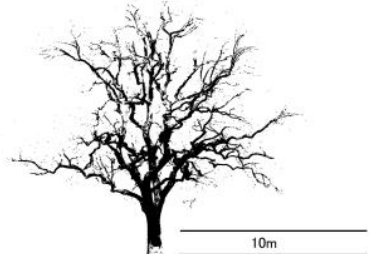

After processing
Figure 7. Noise processing using SOR and MLS

2.1.4 Processing Result of TreeQSM: The trunks and branches point cloud extracted in the previous process were processed by "TreeQSM". The 3D tree models for each tree species are shown in Figure 8, and the whole area is shown in Figure 9.

\section{ACCURACY VERIFICATION OF 3D TREE MODEL}

\subsection{Accuracy verification of 3D tree model constructed by TLS-QSM method}

The TLS-QSM method enables detailed and efficient 3D modeling from tree point cloud data. However, it is not clear how accurate the 3D tree model constructed from the tree point cloud will be. Therefore, the accuracy of the reproducibility of the 3D tree model was clarified by the k-neighborhood algorithm (KNN).

As a method to calculate the accuracy using $\mathrm{KNN}$, we extracted the positional coordinates as a query point for nearest neighbor search from the constructed 3D tree model (Figure 10). After that, using the extracted positional coordinates as a query, a KNN search (set value: $\mathrm{k}=5$ ) was performed on the tree point cloud, and the extent of the 3D tree model constructed from the tree point cloud was clarified. As a result, the 3D tree model was constructed within the standard deviation of $0.016 \mathrm{~m}$ from the tree point cloud data (Figure 11). Furthermore, when the target of the accuracy verification was a tree branch, the 3D tree model was constructed within the standard deviation of $0.007 \mathrm{~m}$ (Figure 12). Therefore, the reproducibility of the tree shape by the TreeQSM method was also effective in terms of accuracy.
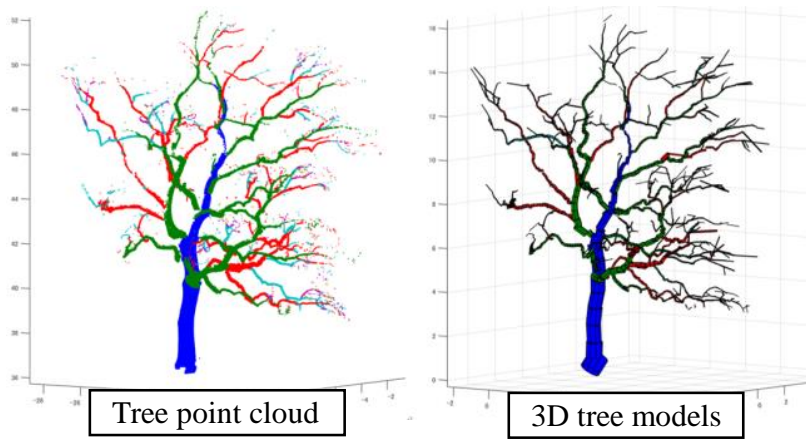

(a) Camphor

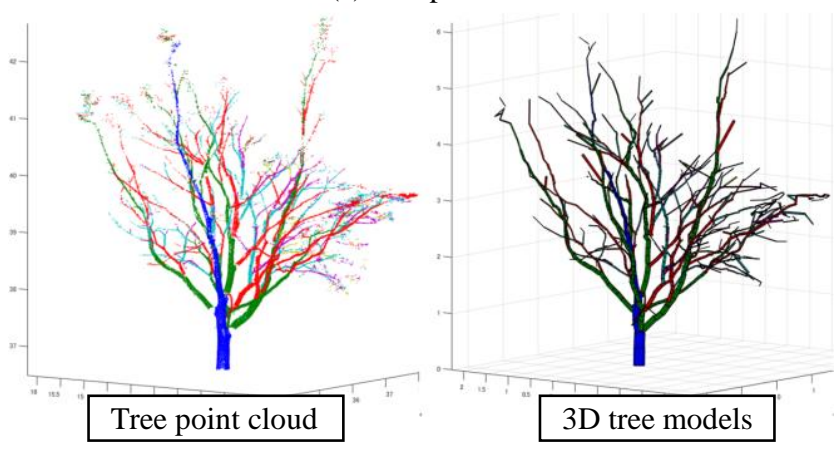

(b) Maple

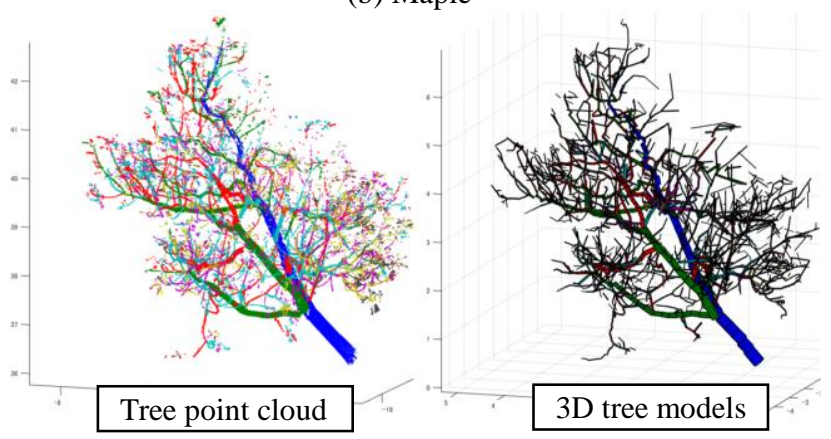

(c) Japanese wax tree

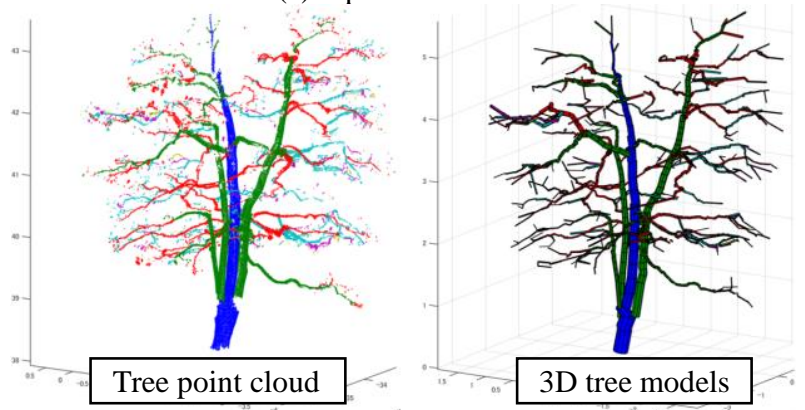

(d) Japanese ternstroemia

Figure 8. 3D tree model for each species

\section{CONCLUSION}

4.1 Importance as technique for preservation and restoration of Japanese garden

A Japanese garden is a space composed mainly of plants that transition and change, and the garden trees are important landscape components that determine the characteristics of Japanese gardens. However, the preservation of garden trees is an important and difficult problem, and the cultural value of Japanese gardens might be lost due to inadequate tree 


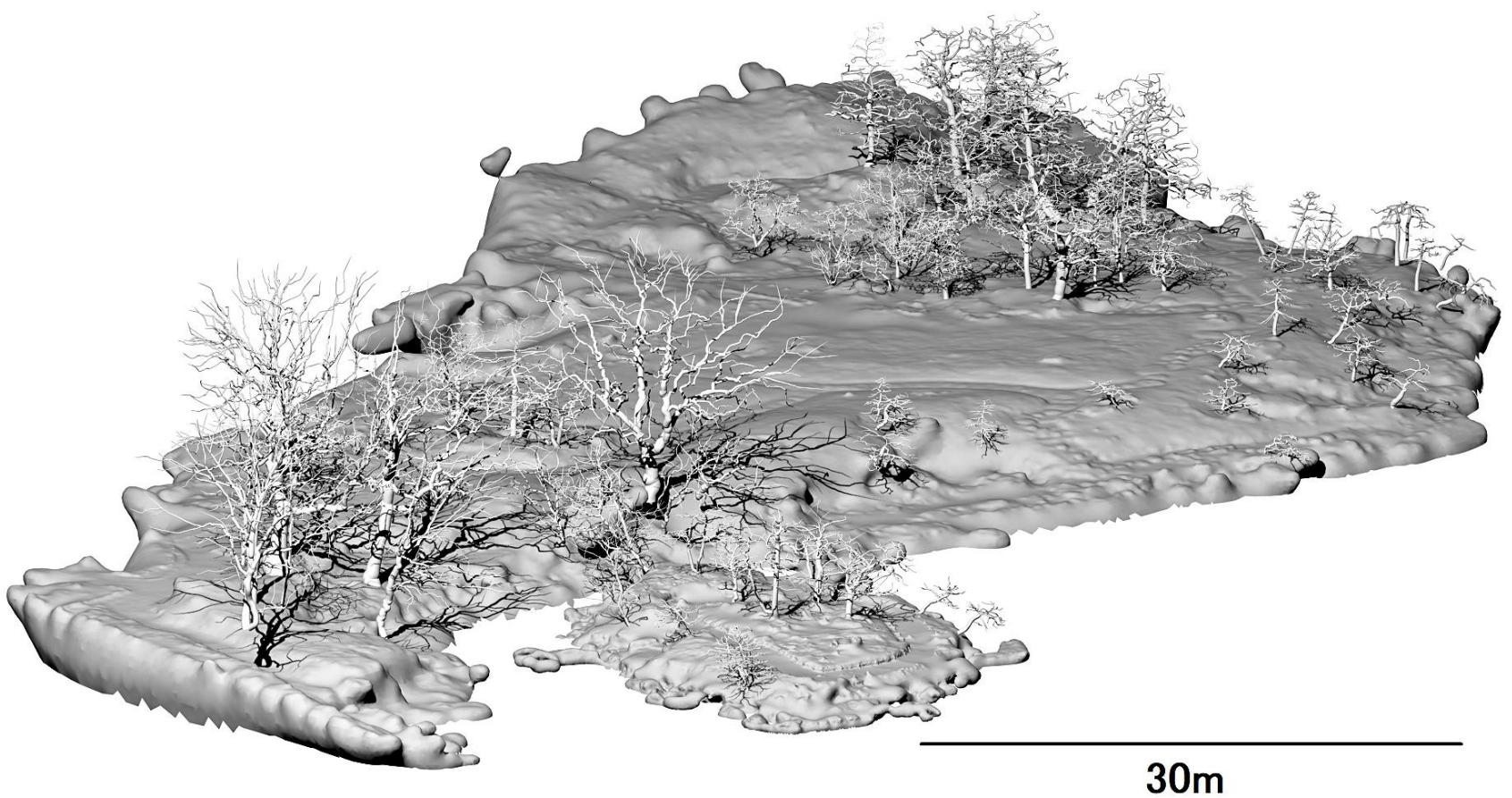

Figure 9. Constructed 3D tree model of the target area

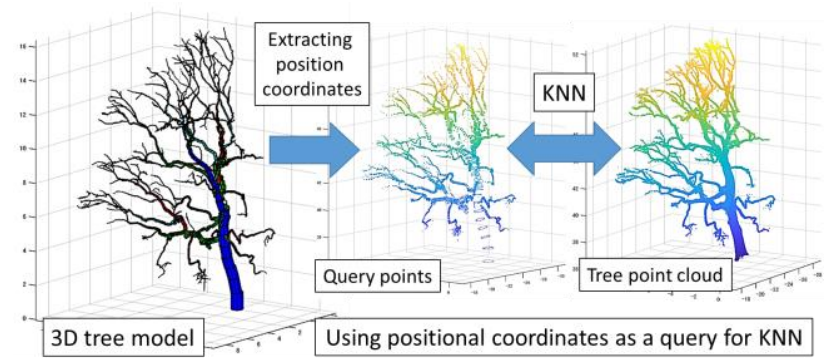

Figure 10. Method of accuracy verification

management. In particular, garden trees that have a significant effect on landscape composition need to have the precision of their tree shape management improved. From this, we clarified that the 3D tree model proposed in this study sufficiently reproduces the tree shape from the main trunk to the trunk/branch on the basis of the results of the accuracy verification, which is important for accurately recording the shape of existing trees and maintaining their shape.

In addition, efforts to restore the lost form of historical Japanese gardens are being actively conducted. However, when restoring the planting, there were few materials that recorded tree shapes of Japanese gardens in their early days in detail, so the planting form were based on old photographs and ezu. Therefore, the 3D tree model generated by our proposed method can also contribute to preserving the landscape of Japanese gardens that existed in that era, especially as a record of the landscape created by the garden trees.

\subsection{Potential for expansion}

The TLS-QSM method used in this study was originally intended to calculate parameters such as the amount of aboveground biomass (AGB) of trees with high accuracy (Gonzalez de Tanago, 2018)(Disney, 2018). Therefore, in addition to the

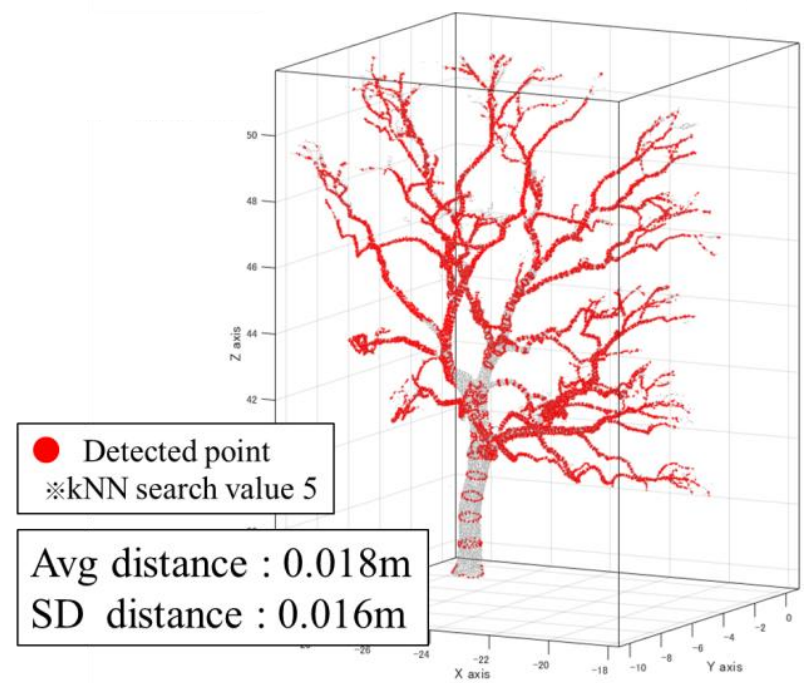

Figure 11. Whole tree accuracy results

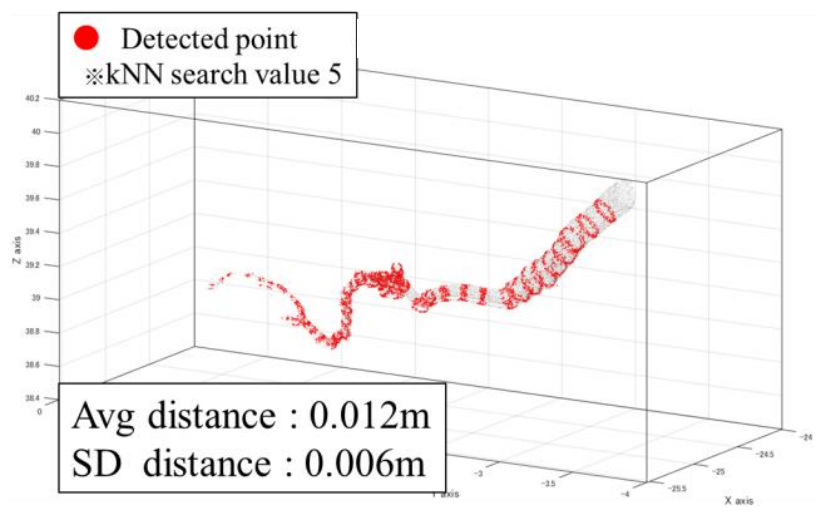

Figure 12. Part of branches accuracy results 
accurate reconstruction of the tree shape disclosed in this study, it is possible to accurately acquire information such as the total volume, trunk volume, branch volume, height, trunk length, and branch length of a tree. Furthermore, the use of the reflectance of the adjunct information may enable detection of withered branches caused by hydropenia. From this, it is possible to acquire parameters to predict the growth of a tree by periodic measurement, to diagnose garden tree activity, and predict changes due to changes in Japanese gardens.

In this study, the importance of a detailed 3D tree model was described mainly from the perspective of landscape architecture. However, detailed 3D tree models are also applicable in various fields such as botany, silviculture, design studies, and climatology, and are said to be important as analytical research materials (Hu, 2017)(Landes, 2015)(Bournez, 2017). Therefore, the construction, utilization, and analysis of $3 \mathrm{D}$ tree models using a TLS is extremely versatile and practical, and directly contribute to society.

\section{REFERENCES}

Åkerblom, M., Raumonen, P., Kaasalainen, M. \& Casella, E., 2015: Analysis of geometric primitives in quantitative structure models of tree stems. Remote Sens. 7, 4581-4603.

Åkerblom, M., Raumonen, P., Mäkipää, R. \& Kaasalainen, M., 2017: Automatic tree species recognition with quantitative structure models. Remote Sens. Environ. 191, 1-12.

Bournez, E., Landes, T., Saudreau, M., Kastendeuch, P. \& Najjar, G., 2017: From TLS point clouds to 3D models of trees: A comparison of existing algorithms for 3D tree reconstruction. Int. Arch. Photogramm. Remote Sens. Spat. Inf. Sci. - ISPRS Arch. 42, 113-120.

Calders, K., et al., 2017: Evaluation of the range accuracy and the radiometric calibration of multiple terrestrial laser scanning instruments for data interoperability. IEEE Trans. Geosci. Remote Sens. 55, 2716-2724.

Computree group, 2010. Computree Forest inventory with Lidar (ONF). Computree, Version 5.0.215c. http://computree.onf.fr/?lang=en (4 May 2019).

Disney, M. I., et al., 2018: Weighing trees with lasers: Advances, challenges and opportunities. Interface Focus 8.

Gonzalez de Tanago, J., et al., 2018: Estimation of aboveground biomass of large tropical trees with terrestrial LiDAR. Methods Ecol. Evol. 9, 223-234.

Hackenberg, J., Spiecker, H., Calders, K., Disney, M. \& Raumonen, P., 2015: SimpleTree - An efficient open source tool to build tree models from TLS clouds. Forests 6, 42454294.

Hu, S., Li, Z., Zhang, Z., He, D. \& Wimmer, M., 2017: Efficient tree modeling from airborne LiDAR point clouds. Comput. Graph. 67, 1-13.

Inverse problems research group at Tampere University, 2019. Inverse Tampere. TreeQSM, Version 2.3.2, GitHub. https://github.com/InverseTampere/TreeQSM (8 January 2019).
Jucker, T., et al., 2017: Allometric equations for integrating remote sensing imagery into forest monitoring programmes. Glob. Chang. Biol. 23, 177-190.

Landes, T., et al., 2015: 3D tree architecture modeling from laser scanning for urban microclimate study. 9th Int. Conf. Urban Clim. 12th Symp. Urban Environ.

Livny, Y., et al., 2010: Automatic Reconstruction of Tree Skeletal Structures from Point Clouds. ACM Trans. Graph. 29, $1-8$.

Raumonen, P. et al., 2013: Fast automatic precision tree models from terrestrial laser scanner data. Remote Sens. 5, 491-520.

Raumonen, P., 2017: TreeQSM Quantitative Structure Models of Single Trees from Laser Scanner Data. Inverse problems research group at Tampere University, Inverse Tampere.

RIEGL Laser Measurement Systems GmbH, 2017. LAS Extrabytes Implementation in RIEGL Software.

Taketoshi, K., 1984: Garden planting designs shown in painting scrolls during the Heian and Kamakura eras. Journal of the Japanese Institute of Landscape Architects 48(5), 67-72. doi.org/10.5632/jila1934.48.5_67.

Taketoshi, K., 1994: A study on the maintenance of garden planting in the Heian and Kamakura period. Journal of the Japanese Institute of Landscape Architects 57(5), 13-18. doi.org/10.5632/jila1934.57.5_13.

Tomoki, K., 2011: Historical changes and restorationconsideration in the kaiusou garden. Journal of The Academic Society of Japanese Garden 25 2011, 23-29. Doi.org/10.5982/jgarden.2011.25_23.

Yasuhiro, A., Katsuhiro, K., 2006: Study on Generation of Tree Shapes with Specific Features of Species Based on a Statistical Method. The IEICE transactions on information and systems (Japanese edition) 89(5), 1019-1035.

Zhang, X., Li, H., Dai, M., Ma, W. \& Quan, L., 2014: Datadriven synthetic modeling of trees. IEEE Trans. Vis. Comput. Graph. 20, 1214-1226. 